\title{
LEO EN LA PRÁCTICA: LA EXPERIENCIA FORMATIVA EN UN CENTRO DE LECTURAS, ESCRITURAS Y ORALIDADES
}

\author{
LEO NA PRÁTICA: A EXPERIÊNCIA EdUCATIVA NUM CENTRO DE LEITURAS, ESCRITAS \\ E ORALIDADES
}

leos in Practice - The Educational Experience in a Center for Readings, SCRibalities, AND Oralities

\section{Luanda Rejane Soares Sito}

Doctora en Lingüística Aplicada, Universidade Estadual de Campinas, Brasil. Profesora e investigadora de la Facultad de Educación, Universidad de Antioquia. Líder del grupo de investigación Educación y Diversidad Internacional (EDI). Integrante de la coordinación colegiada del CLEO - Centro de Lecturas, Escrituras y Oralidades de la Universidad de Antioquia, UdeA. Facultad de Educación, Universidad de Antioquia, Calle 70 N. ${ }^{\circ}$ 52-21, Medellín, Colombia.

luanda.soares@udea.edu.co https://orcid.

org/0000-0001-7579-4229

\section{Juan Camilo Méndez Rendón Doctor en Filosofía, Universidad Pontificia Bolivariana, Medellín.} Magíster en Lingüística, Universidad de Antioquia. Profesor de la Facultad de Educación y Escuela de Nutrición y Dietética, Universidad de Antioquia. Docente del Diploma Lenguaje y Permanencia del CLEO. Miembro de la coordinación colegiada del CLEO. Facultad de Educación, Universidad de Antioquia, Calle 70 N. ${ }^{\circ}$ 52-21, Medellín, Colombia. camilo.mendez@udea.edu.co

\section{RESUMEN}

En este texto, analizamos el diseño y la creación del Centro de Lecturas, Escrituras y Oralidades (CLEO) de la Universidad de Antioquia (UdeA) como un dispositivo de esta universidad pública colombiana para fortalecer las prácticas letradas académicas y apoyar procesos de graduación exitosos. Este centro se consolidó a partir de aportes de enfoques como los Estudios de Literacidad y Alfabetización Académica. Al presentar esta experiencia pedagógica, buscamos mostrar la generalidad del centro, y analizar el funcionamiento de tres estrategias formativopedagógicas en las cuales se sustenta el trabajo del CLEO, sus enfoques y diseño, y los alcances que han tenido en diferentes actores de la comunidad universitaria (docentes, estudiantes y administrativos), con el fin de posicionar como discusión y como reflexión el papel del lenguaje en la vida académica. Además, mostramos cómo estas estrategias contribuyen de diferentes formas al proceso formativo de los estudiantes universitarios. Como consideraciones, señalamos que quizá este centro haga parte de una segunda generación de centros de lectura y escritura, al pluralizar su visión de lenguaje y orientarse a perspectivas interculturales, al tiempo que considera la oralidad en sus múltiples y diversas ocurrencias.

Palabras clave: centro de lectura y escritura, educación superior, literacidades, alfabetización académica, formación.

\section{Resumo}

Neste artigo, analisamos o desenho e a criação do Centro de Leitura, Escrita e Oralidade (CLEO) da Universidade de Antioquia (UdeA) como um dispositivo dessa universidade pública colombiana visando fortalecer as práticas de letramento acadêmico e apoiar processos de graduação bem-sucedidos. Este centro foi consolidado com base em contribuições dos Estudos de Letramento e de Alfabetização Acadêmica. Ao presentar esta experiência pedagógica, buscase analisar o funcionamento de três estratégias formativo-pedagógicas nas quais se baseia o trabalho de CLEO, suas abordagens e desenho, e os alcances que eles tiveram em diferentes atores na comunidade universitária (professores, alunos e administrativos), a fim de posicionar como discussão e reflexão o

Recibido: 2018-11-16 / Aceptado: 2019-03-06 / Publicado: 2019-05-20

DOI: $10.17533 /$ udea.ikala.v24n02a12

Íkala, Revista de Cenguage y Cultura

Medellín, Colombia, Vol. 24, Issue 2 (May-August, 2019), pp. 419-438, ISSN 0123-3432 
Leidy Yaneth Vásquez Ramírez Candidata a doctora en Educación, Universidad San Buenaventura, Medellín. Magíster en Educación. Especialista en Literatura con Énfasis en Producción de Textos e Hipertextos, Universidad Pontificia Bolivariana, Medellín. Licenciada en Educación Básica con énfasis en Humanidades: Lengua Castellana, Universidad de Antioquia.

Docente del Diploma Lenguaje y Permanencia del CLEO. Miembro de la coordinación colegiada del CLEO. Facultad de Educación, Universidad de Antioquia, Calle 70 N. ${ }^{\circ}$ 52-21, Medellín, Colombia. lyaneth.vasquez@udea.edu.co https://orcid. org/0000-0002-1090-7234 papel da linguagem na vida acadêmica. Além disso, mostramos como essas estratégias contribuem de diferentes maneiras para o processo de formação dos universitários. Como considerações, ressaltamos que este centro pode fazer parte de uma segunda geração de centros de leitura e escrita, ao pluralizar sua visão de linguagem e orientar-se para perspectivas interculturais, considerando a oralidade em suas múltiplas e diversas ocorrências.

Palavras-chave: centro de leitura e escrita; ensino superior; letramento; alfabetização acadêmica; formação.

\section{Abstract}

In this paper we will examine the setting up of the Reading, Writing and Orality Center (CLEO) at University of Antioquia (UdeA) as a device through which this Colombian public university is seeking to strengthen academic literacy practices and supporting successful graduation processes. This Center relies upon contributions from Literacy and Academic Literacy Studies. In presenting this pedagogical experience, we aim to present an overview of the Center, and to analyze the workings of three educational-pedagogical strategies on which the work of CLEO is based, its approaches and design, and its impact in different university community actors (teachers, students, and administrative staff). This will help us discuss and reflect upon the role of language in academic life. Additionally, we will show how these strategies contribute in different ways to university students' formation. We also we point out that this Center might be a part of a second generation of Reading and Writing Centers, since it pluralizes its language approach and addresses intercultural perspectives, including studying orality in its manifold and varied occurrences.

Keywords: reading and writing center; higher education; literacies; academic literacy; training. 


\section{Introducción: ¿ Por qué un centro de lectura, escritura y oralidad?}

La iniciativa de conformar un centro de lectura, escritura y oralidad se inició entre los años 2011 y 2015 con propuestas de prácticas docentes en la Facultad de Educación, como una forma de jalonar procesos de acompañamientos entre pares. ${ }^{1}$ Más adelante se extendió a otras dependencias académicas, hasta integrar una mesa inicial de asesores conformada por la Facultad de Comunicación, la Escuela Interamericana de Bibliotecología, la Escuela de Idiomas, la Facultad de Artes y la Facultad de Educación.

La reunión de participantes de estas unidades académicas supuso, a su vez, un encuentro de posturas, enfoques, comprensiones y prácticas alrededor del lenguaje, situación que no estuvo exenta de las tensiones propias del entrecruzamiento de ideas sobre este tema. Sin embargo, esta heterogeneidad permitió incluir en las comprensiones y en las prácticas del CLEO una mirada amplia sobre los usos que recibe el lenguaje en la educación superior y los actores que lo habitan. De ahí que hablemos de un centro de Lecturas, Escrituras y Oralidades (LEO) en plural, como un espacio de lenguajes que se ponen en diálogo, pero también en tensión.

Desde el año 2015, el plan de fomento a la calidad, en su línea de permanencia estudiantil, ${ }^{2}$ viene

1 Se refiere a estudiantes que están en el último tramo de su formación en pregrado. Se caracterizan por su alto rendimiento académico y por su disposición para asesorar y acompañar procesos de apropiación discursivos desde los géneros académicos con otros estudiantes de pregrado, de ahí el nombre de pares.

2 El plan de Fomento a la Calidad es una iniciativa realizada con recursos provenientes del impuesto sobre la renta para la equidad (CREE) del Ministerio de Educación Nacional de Colombia, que se propone a fortalecer procesos institucionales y dejar capacidades instaladas, por medio de proyectos, iniciativas y acciones, para la mejora de la calidad académica y administrativa de la Universidad de Antioquia. Su meta principal es fomentar el acceso, la permanencia y la graduación estudiantil. Ver preguntándose por el vínculo entre lenguaje y permanencia. Para responder esta pregunta, se planteó la tarea de configurar un dispositivo institucional que pusiera en diálogo las iniciativas que las diferentes unidades académicas han llevado a cabo en el pasado o están adelantando actualmente, y se agrupara en un centro el abordaje de las prácticas de lecturas, escrituras y oralidades, con el ánimo de fomentar la permanencia estudiantil en la Universidad de Antioquia. Así surgió el CLEO, como un dispositivo institucional que fortaleciera los procesos de revisión y atención de las prácticas LEO en educación superior y por el interés institucional de reconocer factores académicos que limitan el acceso a la universidad, la permanencia en el claustro y la graduación estudiantil.

El CLEO se inscribe en una tradición de centros que busca no solo entender un estado de cosas, sino también aportar elementos para su transformación, con el ánimo de mejorar la calidad de vida de los estudiantes. En este sentido, el objetivo del CLEO es fortalecer procesos académicos en torno a la lectura, la escritura y la oralidad que propicien la calidad, la continuidad y la culminación exitosa de los programas de formación que adelantan los estudiantes de la Universidad de Antioquia.

Así, al presentar esta experiencia pedagógica, buscamos mostrar la generalidad del centro, y analizar el funcionamiento de tres estrategias formativopedagógicas en las cuales se sustenta el trabajo del CLEO, sus enfoques y diseño, y el impacto que ha tenido en diferentes actores de la comunidad universitaria (docentes, estudiantes y administrativos). Para ello, dividimos este artículo en cuatro momentos: en el primero, presentamos el centro y las causas de su creación; a continuación, explicamos los enfoques teóricos que subyacen a las acciones de este dispositivo que busca fortalecer las prácticas letradas académicas y apoyar procesos exitosos de graduación; seguimos con una descripción y análisis de tres estrategias

más en: http://www.udea.edu.co/wps/portal/udea/ web/inicio/institucional/permanencia-estudiantil 
formativo-pedagógicas diseñadas y ofrecidas por el CLEO; y cerramos con algunas consideraciones finales.

\section{Presentación del centro}

Empezamos por hacer un breve recorrido por los hitos que han marcado la historia del CLEO, entre 2015 y 2019, de forma que podamos ver cómo se estructuró el centro desde el principio como una comunidad de diálogo, a partir de cinco estrategias y una convocatoria para el acompañamiento a las iniciativas que se venían realizando en la UdeA en torno a las prácticas de lenguaje, vinculadas, solo en algunos casos, con la pregunta por la permanencia. De esta manera, en el año 2018, el CLEO empezó a institucionalizar las acciones de trabajo mediante la consolidación del dispositivo. Este trayecto se evidencia de manera sintética en la Figura 1, que le sirve al equipo base para pensar en las proyecciones de continuidad, evaluación y reconfiguración 422 de sus acciones a mediano y largo plazo.

Como lo muestra la imagen, el CLEO es un dispositivo construido de manera colaborativa, pues surge de las iniciativas de maestros y estudiantes de diferentes unidades académicas, en el convencimiento de que el lenguaje moviliza acciones y genera posibilidades de permanencia y mejores trayectos universitarios para los profesionales en formación de la U. de A. CLEO se plantea una pregunta por el lenguaje como dispositivo de poder que favorece o dificulta el acercamiento de los estudiantes a sus procesos formativos vinculados con disciplinas específicas. Para el año 2016, nos proponemos un ejercicio arqueológico que nos permitiera ver qué se hace y cómo se llevan a cabo las prácticas de Lectura, Escritura y Oralidad en la UdeA en la formación académica. Para esto invitamos a la comunidad universitaria a tomar partido a través de estrategias de docencia y de investigación que se llevaron a cabo durante el año 2017 y que nos permitieron derivar en la consolidación del CLEO como proyecto de complejidad institucional, que en el año 2019 sigue mirando al futuro con el ánimo de apostarle a la permanencia, la continuidad y la culminación exitosa de los trayectos formativos de nuestros estudiantes, desde diálogos entre los discursos teóricos y prácticos de las LEO en el contexto universitario.

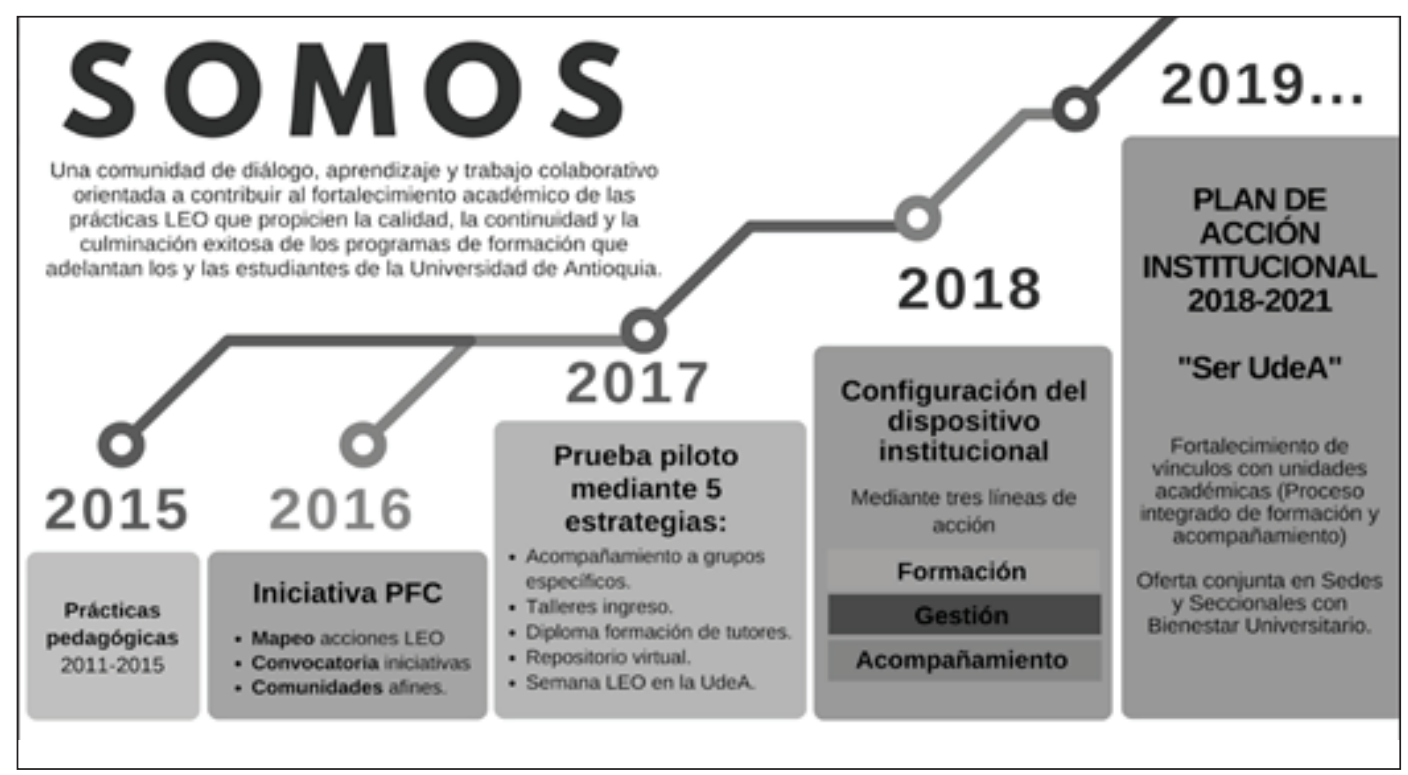

Figura 1 Trayecto del CLEo 2015-2019. 
El diseño del CLEO, para el 2018, se ha configurado a partir de estrategias enmarcadas en tres líneas de acción: gestión, formación y acompañamiento, así:

- Línea de gestión: su objetivo es fortalecer aspectos de la gestión académica, administrativo-financiera y comunitaria que hacen posible los procesos de formación y acompañamiento CLEO.

- Línea formación: tiene como objetivo formar tutores para el fortalecimiento de la permanencia estudiantil y el mejoramiento de la calidad académica en relación con las prácticas LEO.

- Línea acompañamiento: mediante la cual se buscan generar procesos de acompañamiento formativo en lectura, escritura y oralidad, tanto a estudiantes de pregrado, posgrado, como a docentes de las diferentes Unidades Académicas de la Universidad de Antioquia (Figura 2).

Estas líneas trabajan de modo articulado con base en tres grandes principios: a) una orientación de las propuestas del CLEO hacia la diversidad cultural y lingüística, b) un reconocimiento de que los diferentes lenguajes inciden en la permanencia estudiantil: las prácticas LEO son claves para el ingreso a la cultura académica universitaria, y c) una búsqueda por visibilizar las maneras de usar los lenguajes y lenguas que habitan la universidad: lenguajes expresivos y estéticos, lengua de señas, lengua castellana, lenguas ancestrales y lenguas extranjeras (mirada plurilingüe). En síntesis, estos principios del CLEO nos invitan a valorar y poner en práctica una apuesta formativa con: la permanencia como propósito central (articulando lenguaje, enseñanza y aprendizaje y políticas LEO); una perspectiva plurilingüe (poniendo en diálogo lenguajes inclusivos, lenguajes expresivos y estéticos, lengua de señas, lenguas ancestrales, lengua castellana); y una perspectiva crítica e intercultural (aportes de las voces del Sur para el reconocimiento del otro).

La comunidad del CLEO ha crecido en relaciones, iniciativas, estrategias, e integrantes en este camino de encuentros, cooperación y aprendizajes; y sus miembros a su vez han afianzado su participación en proyectos académicos, consolidando propuestas - que antes eran planes y que cada vez se vuelven más tangibles con la creación

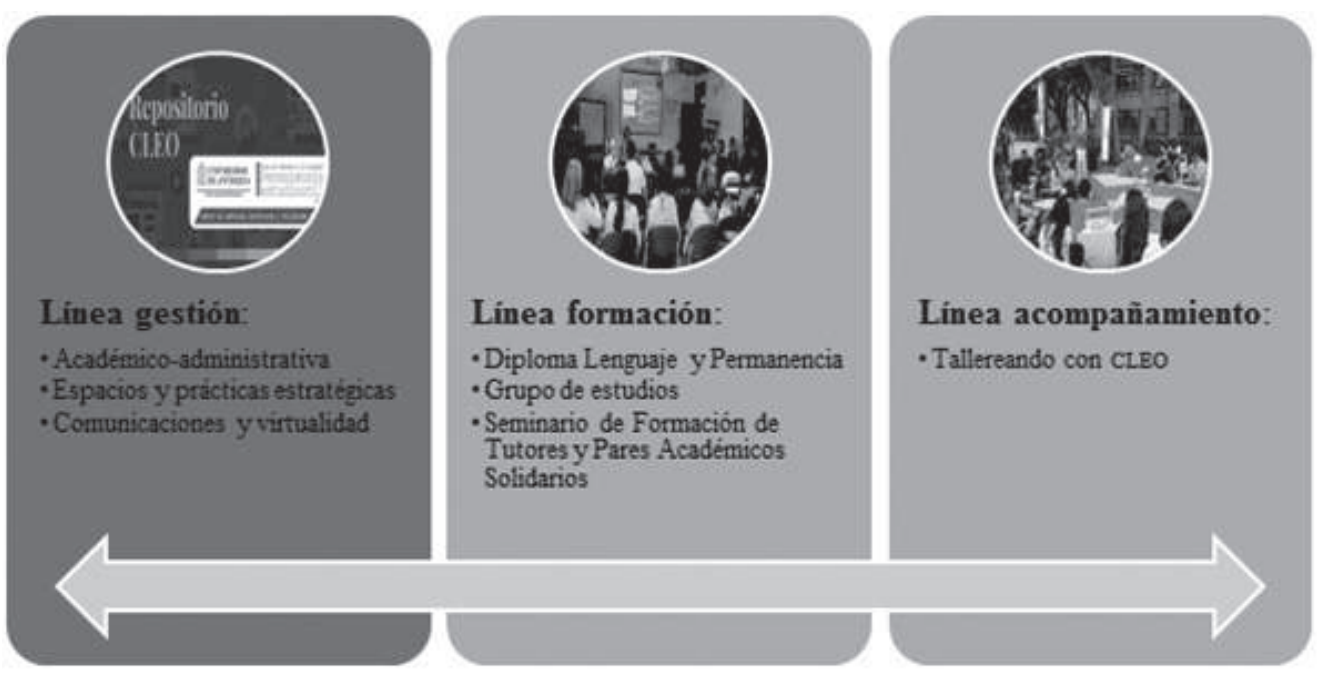

Figura 2 Líneas de trabajo del CLEO. Fotos: archivo CLEO. 
de grupos de trabajo - así como de una comunidad que quiere pensar el papel del lenguaje en la vida universitaria. En consonancia con lo anterior, para 2019 esperamos mantener nuestra oferta académica siguiendo los objetivos del Plan de acción institucional (2018-2021) de la UdeA, bajo la dirección de la Vicerrectoría de Docencia.

Esta apuestadel CLEO, consideramos, escongruente con la misión de la Universidad de Antioquia. Ya dijimos cómo en el CLEO confluyen diferentes usos del lenguaje en las comunidades académicas y universitarias. Tales usos, sin embargo, son comprendidos desde los presupuestos de las literacidades críticas, entendiendo el lenguaje como una construcción social, producto de una época y una circunstancia histórica, que lo reconfigura y lo dota de intencionalidades. En este objetivo, refleja la misión de la universidad y el compromiso de la educación pública:

La Universidad de Antioquia, patrimonio científi-

co, cultural e histórico de la comunidad antioqueña y nacional, es una institución estatal que desarrolla el servicio público de la educación estatal con criterios de excelencia académica, ética y responsabilidad social. En ejercicio de la autonomía universitaria, de las libertades de enseñanza, aprendizaje, investigación y cátedra que garantiza la Constitución Política, y abierta a todas las corrientes del pensamiento cumple, mediante la investigación, la docencia y la extensión, la misión de actuar como centro de creación, preservación, transmisión y difusión del conocimiento y de la cultura. (UdeA, 2015, los resaltados son nuestros)

En los destacados de la cita, queremos resaltar dos cosas: la primera es hacer ostensible la coherencia interna que un centro como el CLEO debe guardar con los lineamientos teleológicos de la institución a la que pertenece, y la segunda tiene que ver con el compromiso con lo público, que lleva a buscar que las acciones del CLEO trasciendan la vida universitaria.

Luego de esta parte introductoria, en la cual presentamos los lineamientos que guiaron la creación del Centro de Lecturas, Escrituras y Oralidades (CLEO) de la Universidad de Antioquia (UdeA), presentamos a continuación un apartado sobre los enfoques del lenguaje que orientan las acciones formativas del centro. Con base en ellos buscamos presentar las LEO como prácticas pertinentes y viables para incorporarse a los diferentes programas curriculares que se desarrollan en la universidad.

\section{Un espacio de conversación: perspectiva sociocultural y posturas del CLEO}

En esta sección, buscamos explicitar los enfoques teóricos que subyacen a las acciones de este dispositivo que busca fortalecer las prácticas letradas académicas y apoyar procesos de graduación exitosos. Para ello, debemos comenzar aclarando que hay diferentes campos del conocimiento que se ocupan de la lectura y la escritura en la educación superior. Durante el "IV Writing Research Across Borders (WRAB)/ Investigación en Escritura a través de las Fronteras"3 (2017), por ejemplo, se pudo observar que diferentes disciplinas vienen (pre)ocupándose cada vez más de los procesos de lectura y escritura en la formación. Así, entre los participantes, hubo investigadores de las áreas de lingüística, lingüística aplicada, educación, así como de ingeniería, enfermería, entre otras. Todos indagando por el lugar de la escritura en su formación profesional.

En el escenario latinoamericano, la investigación sobre la lectura y la escritura en la educación superior es reciente. Como lo muestra Carlino (2013), este tema apenas emerge a inicios del siglo XXI, tanto en el contexto hispanohablante como en contextos lusohablantes. En este escenario, el tema de la escritura en la educación superior llega en medio de un discurso de "crisis", como analizan Zavala y Córdoba (2010), en el cual muchas veces se estigmatiza o culpabiliza a los estudiantes por "no saber leer ni escribir", expresión con la cual juega Soler (2013) para desvelar esta realidad. Para ilustrar las perspectivas teóricas que en mayor

3 El IV Writing Research Across Borders (WRAB)/ Investigación en escritura a través de las fronteras, se realizó del 15 al 18 de febrero de 2017, en la Pontificia Universidad Javeriana, de Bogotá, Colombia. 
o menor medida se oponen a esta idea de crisis hablaremos de los campos de alfabetización académica y literacidad académica, ${ }^{4}$ los cuales vienen desarrollando un gran conjunto de investigaciones que, por su carácter sociocultural o sociorretórico, contribuyen a una formación más plural de estudiantes y maestros.

\section{Una comparación entre alfabetización académica y literacidad académica}

El centro inició su trabajo revisando programas ya implementados en la institución, para aprovechar el potencial institucional. Su objetivo era conformar una comunidad académica en torno al tema de lenguajes para la permanencia y dejar capacidad instalada en sus unidades académicas. Este punto de partida responde a los enfoques teóricos que subyacen al diseño de las estrategias formativo-pedagógicas en las cuales se sustenta el trabajo del CLEO. Sus enfoques y el diseño correspondiente han entablado un diálogo con los campos de la alfabetización académica y la literacidad académica, que estaban en desarrollo en las líneas de prácticas de programas en la Facultad de Educación de la UdeA. También cabe mencionar que la elección de estos enfoques está alineada con una tendencia internacional, como señala Moreno (2019), en una revisión del estado del arte.

En el primer enfoque - alfabetización académica-, la gran exponente en Latinoamérica es la investigadora argentina Paula Carlino. En su trabajo, ha traducido y reseñado una serie de estudios

4 Es importante señalar, como destaca Moreno (2019) en una revisión del estado del arte del campo, que hay diferentes tendencias emergentes en el campo de las prácticas de lectura, escritura y oralidad en la Universidad. La autora destaca líneas como "Escribir a través del currículum" (Bazerman et al., 2016), "enseñar escritura en contexto" (Chalmers y Fuller, 1996), alfabetización académica (Carlino, 2005), literacidad académica (Zavala, 2011; Kleiman, 1995, Lea y Street, 1998, Sito y Kleiman, 2017), literacidad disciplinaria (Moje, 2008; Moreno, 2019) y función epistémica de la lectura. Por cuenta del objetivo de este artículo, nos centraremos en los aportes que más han influenciado las estrategias del CLEO. y experiencias con la escritura académica, especialmente en el contexto anglosajón. Carlino (2002, 2013) reitera la preocupación didáctica y educativa de este campo, indagando siempre por los procesosde enseñanza y aprendizaje profesionales mediados por la escritura, así como ejemplificando formas de contemplarlos en el aula universitaria.

Para la autora, es clave entender que "aprender los contenidos de una materia consiste en una tarea doble: apropiarse de su sistema conceptual-metodológico y también de sus prácticas discursivas características, ya que una disciplina es un espacio discursivo y retórico, tanto como conceptual" (Carlino, 2002, p. 7). Al comprender esta complejidad, la autora advierte que enseñar los modos de leer y escribir en la disciplina debe ser llevada a cabo por los maestros del área, ya que son ellos los que más conocen sus prácticas discursivas, a la par que "están familiarizados con el contenido difícil que los estudiantes tratan de dominar" (p.7).

En su recorrido teórico-investigativo, Carlino recoge una serie de propuestas didácticas que pueden fomentar ideas para aprovechar la dimensión epistémica de la escritura en el aula, pues fortalecerían el repertorio lingüístico de los estudiantes para que participen de modo más exitoso en sus campos de formación. Este enfoque nos lleva a pensar estrategias orientadas a los diferentes públicos de la institución universitaria, pues se asume que la responsabilidad por las prácticas de lectura, escritura y oralidad debe ser institucional también, y no apenas de los estudiantes. Los expertos en sus campos tienen mucho que aportar en la apropiación de las convenciones académicas de sus estudiantes; por ello, el rol de los centros puede expandirse a pensar en la formación de docentes y administrativos para que reconozcan el lugar del lenguaje en la formación universitaria.

Esta postura didáctico-educativa se enriquece con una mirada más lingüístico-etnográfica, para entender cómo la gente usa la escritura. Así, en el segundo enfoque — literacidad académica—se busca revisar dentro de un marco crítico el porqué 
de los discursos de crisis sobre el uso de la escritura en la universidad. En muchos espacios del mundo hispanohablante y también en Brasil, la vertiente sociocultural de los estudios de literacidad, o letramento (Heath, 1982; Street, 1993; Kleiman, 1995; Zavala, Niño-Murcia y Ames, 2004; Kalman y Street, 2009), consolidada desde los años 1990, centra su atención en los procesos interaccionales entre los sujetos, y busca comprender cómo las personas se apropian de la escritura, así como cuáles son los contextos en que el uso de la escritura ocurre.

Sobre este piso teórico, el campo de la literacidad académica (Zavala y Córdoba, 2010; Zavala, 2011) emergió, en los últimos cinco años, anclado a los estudios de literacidad, área que ocupa un lugar destacado en la lingüística aplicada, donde surgieron por las razones ya descritas, y también como un intento de separar los estudios sobre "el impacto social de la escritura" de los estudios sobre alfabetización y habilidades cognitivas involucradas en la lectura y escritura.

Un ejemplo de los estudios de literacidad en la esfera académica es el estudio de Zavala y Córdoba (2010) sobre los conflictos que vivencian jóvenes universitarios quechuahablantes al lidiar con las prácticas letradas académicas. La ponderación de las autoras sobre esa relación de estudiantes universitarios con la escritura académica amplía el tema, al puntualizar que

los conflictos que emergen entre estudiantes y docentes con relación a la lectura y la escritura en la universidad no se restringen simplemente a la técnica de la lectura y la escritura, a las habilidades o a la gramática sino a aspectos que están relacionados con la identidad, la epistemología y el poder. Esto quiere decir que la lectura y la escritura también se relacionan con nuestro sentido de pertenencia a la comunidad de la que intentamos formar parte, con las maneras de construir conocimiento y con las valoraciones diferenciadas que se adscriben a las diversas formas de lectura y escritura que se practican en nuestra sociedad. (Zavala y Córdoba, 2010, p. 113)
En este planteamiento, las autoras defienden que la comprensión de la lectura y la escritura en la universidad requiere contemplar la aprehensión de aspectos como pertenencia, valores y maneras de construir conocimiento por los sujetos. Esta observación provoca un vuelco en el objeto de los centros de lectura y escritura, pues nos provoca mínimamente en tres aspectos: i) dejar de ver la lengua solo como algo instrumental y neutro; ii) ampliar la visión de lengua a una mirada hacia los lenguajes, y iii) orientarse hacia un marco de plurilingüismo e interculturalidad.

En este enfoque, nociones como prácticas institucionales del misterio (Lillis, 1999), o las dimensiones escondidas (Street, 2010), por ejemplo, describen las formas de hacer y decir (con, por y en el lenguaje) en la universidad y emergen para mostrar que hay mucha invisibilidad sobre las mismas en las instituciones (misterio, escondidas). En su desarrollo conceptual, buscan explicitar las dimensiones veladas que perjudican aún más a aquellos culturalmente distantes de la universidad, pues los modos de hacer y decir en cada contexto tienen sus convenciones, y en la universidad no es diferente. De este modo, aquellos estudiantes que ingresan tienen que ir desvelando poco a poco esas convenciones, y eso, muchas veces, es atravesado por experiencias traumáticas (Sito y Kleiman, 2017; Sito, 2016); a esto se suma que muchas veces las tensiones se reducen a aspectos de la estructura lingüística.

A diferencia de otros contextos, en la discusión hispanohablante algunos cuestionan si los términos alfabetización académica y literacidad académica pertenecen al mismo campo teórico. Para responder esta pregunta, haremos algunas precisiones que tocan ambos conceptos. El campo de alfabetización académica tiene un enfoque más didáctico-educativo, mientras que el campo de la literacidad académica está más enmarcado en un enfoque discursivo-etnográfico. En otras palabras, el primero aborda más las acciones institucionales y didácticas de la enseñanza-aprendizaje en las disciplinas o campos de saber, mientras que el 
segundo se ocupa de entender la participación en culturas escritas en la esfera académica. Además, destacaríamos que, en diálogo con la ponderación que teje Carlino (2013), ambos se configuran en campos diferentes, pues pese a que están articulados, poseen objetos, metodologías y preguntas de investigación alejados.

Otra diferencia de los estudios de literacidad académica, por ejemplo, es que parten de las pedagogías críticas (Freire, 1996; 2012 [1970]), pedagogías culturalmente sensibles (Hornberger, 2001; Ladson-Billings, 2008) y los estudios de literacidad para (re)pensar procesos de enseñanza y aprendizaje en contextos formales y no formales, tomando como presupuesto que pensar en la enseñanza-aprendizaje de la lectura y la escritura no implica solo pensar aspectos de la lengua, sino también contemplar aspectos de la identidad y de las relaciones de poder que atraviesan los usos de lenguaje. ${ }^{5}$ Entretanto, el campo de la alfabetización académica dialoga más con los campos de la didáctica general y las didácticas de las lenguas.

\section{Estudios sobre lectura y escritura académica en Colombia}

En las investigaciones colombianas, el abordaje sociocultural del lenguaje se viene posicionando más recientemente, como lo ilustran varios estudios de la última década (Soler, 2013; Pérez y Rincón, 2013, y Vargas Franco, 2015a). Como antecedentes, el trabajo de Narváez y Cadena

5 En Sito (2014), también hacemos esta distinción: "Podríamos resumir argumentando que, aunque, para algunos investigadores ambos términos nombran el mismo campo teórico, para la investigadora Carlino (2013) conviene distinguir los significados de los dos términos. Para ella, mientras la "alfabetización académica" está orientada para el hacer educativo, o letramento acadêmico enfoca un conjunto de prácticas culturales en torno del uso de textos. Esa distinción también es reiterada por la investigadora Zavala (2013), por entender que ambos términos se refieren a conceptos que poseen objetos de investigación diferentes, luego se trata de categorías, problemas teóricos y apuestas metodológicas diferenciadas, aunque interconectados".
(2008), con un enfoque más lingüístico, ilustra investigaciones que venían indagando por los modos de leer y escribir en la universidad a través de estudios comparativos y análisis de clases.

Pero el estudio de Pérez y Rincón (2013) marca un divisor de aguas en las investigaciones sobre lectura y escritura en la educación superior. Esta investigación de largo aliento reunió diecisiete universidades públicas y privadas de diferentes regiones del país para preguntarse cómo se lee y escribe en las universidades colombianas. También es denso metodológicamente, al articular diferentes metodologías en su desarrollo: encuestas, análisis documental (de programas de cursos y políticas institucionales), grupos de discusión y estudio de caso. El estudio cuanticualitativo revela un mapeo consistente de la situación del lenguaje en la educación superior. Uno de los resultados muestra que las notas de clase son el texto más leído por los estudiantes, mientras hay poca lectura de fuentes primarias. Esta observación indica que la escritura se utiliza en gran medida para reproducir la voz del docente, pero se olvida de fortalecer el uso de la escritura y la lectura en su función epistémica, es decir, leer y escribir para apoyar la formación profesional. Además, el trabajo delineó tres grandes ejes teóricos que enmarcan la investigación en lectura y escritura en el país: 1) la cultura académica, 2) la didáctica de la lengua, y 3 ) las prácticas de lectura y escritura (la perspectiva sociocultural).

A la luz de lo anterior, puede decirse que, en un país como Colombia, el enfoque sociocultural de las LEO ha ganado un espacio en las comprensiones teóricas y en los abordajes de la práctica, tanto en la docencia como en la investigación. Es más, la tendencia determina una distancia en el enfoque con otros países de la región. El estudio transversal de Navarro, Ávila, Tapia-Ladino, Cristovão, Moritz, Narváez, y Bazerman (2016) que cubrió los enfoques de este tipo de estudios en Latinoamérica, encontró que las nociones de proceso y prácticas situadas cobran especial relevancia para el caso colombiano. 
En el corpus colombiano se detecta un interés por la lectura y la escritura como prácticas y procesos situados desde una perspectiva educativa, mientras que los corpora argentino y chileno se destacan por una perspectiva lingüística de la lectura y la escritura como discursos especializados o productos de esos discursos, y el corpus brasileño se ubica en un punto intermedio en este continuo (Navarro et al., 2016).

Lo anterior demuestra hasta qué punto los estudios que se han adelantado en Colombia sobre lectura y escritura en la educación superior se han hecho predominantemente bajo el enfoque de prácticas socioculturales mediadas por los presupuestos teóricos de la literacidad, es decir, que para comprender el desempeño de los estudiantes en prácticas letradas es imprescindible integrar su contexto social y su historia. Con esto, llegamos a dos conclusiones que nos acercan de forma decidida a la postura que el CLEO mantiene sobre el lenguaje. Primero, al considerar que tanto la lectura como la escritura son prácticas situadas y hacen parte de procesos que se extienden en el tiempo, no hay un nivel de escolaridad en el cual dichos procesos culminen: ${ }^{6}$ la formación es continua, inacabada. La segunda conclusión sobre la que queremos llamar la atención es que las prácticas LEO no son inseparables de la pregunta por las condiciones de realización, y tratar de abordarlas de una manera distinta solo atiende a mecanizar, cuando no a instrumentalizar dichas prácticas. Las consecuencias de esto serán visiones esquemáticas sobre la comunicación, cuyas posibilidades no encuentran una forma de proyección más allá del espacio de la escuela o la universidad.

$\mathrm{Al}$ indagar por la trayectoria escolar y universitaria de estudiantes afrodescendientes e indígenas, Soler (2013), desde el análisis crítico del discurso, profundiza en un aspecto relevante de los estudios de literacidad: la diversidad cultural. La autora

6 Vale decir que este es un principio compartido con la alfabetización académica. Al respecto, véase Carlino (2003). parte de una perspectiva histórica y retoma especificidades de la llegada de la escritura española al contexto latinoamericano. Este marco histórico que es discutido por la autora da densidad y profundidad a la comprensión de los enlaces entre lenguaje y poder, ${ }^{7}$ así como permite entender que más allá de la apropiación de las convenciones académicas, muchos estudiantes de comunidades históricamente excluidas del contexto universitario lidian con procesos de resistencia, lucha y exclusión de las representaciones letradas, para luego de duros trayectos de formación lograr entrar en una cierta "armonía" con la identidad universitaria.

Estos estudios pueden reforzarse con el abordaje de Vargas Franco (2015a; 2015b), quien pone en diálogo los estudios de literacidad y el tema de la tecnología digital. Su experiencia en cursos de escritura de géneros académicos genera nuevas preguntas al indagar por el contexto actual de presencia de las TIC en la cotidianidad de los estudiantes universitarios.

Como se observa del recorrido anterior, las prácticas de lectura y escritura - y la oralidad, en la perspectiva sociocultural - vienen siendo analizadas en diferentes campos. Dentro de los estudios lingüísticos, destacamos los estudios de literacidad académica, la escritura a través del currículo (o su sigla en inglés WAC), los estudios sobre los géneros discursivos y la alfabetización académica. Sin embargo, infelizmente, no siempre estos campos nutren los trabajos realizados en los centros de

7 La perspectiva histórica es una dimensión sine qua non para comprender las relaciones de poder establecidas históricamente y las categorías de "diversidad" que generan desigualdad en las sociedades contemporáneas. Un ejemplo es el trabajo de Soler y Pardo (2007), en el cual se desarrolla un consistente análisis de las estrategias discursivas en los medios y en textos escolares, describiendo los modos de funcionamiento del racismo en Colombia; así como su tensión entre la invisibilidad y la exclusión de los grupos afrocolombianos e indígenas. Las propuestas educativas, si quieren construir justicia social, necesitan dialogar más con trabajos críticos y de abordajes más descriptivos, discursivos y etnográficos. 
lectura y escritura en las universidades, tal y como se muestra a continuación.

\section{Los centros de lectura y escritura académica universitarios}

Uno de los dispositivos más extendidos para acompañar los procesos de lectura y escritura de los estudiantes en las universidades son los centros de escritura. De una marcada tradición norteamericana, estos centros abordaron, en un comienzo, funciones remediales que garantizaran la correcta adecuación de los procesos de lectura y escritura a los requerimientos de la educación superior. En este sentido, el papel del tutor responde más a la necesidad de un "acompañante" o de un asesor, que al de un formador.

Aunque los orígenes de los centros responden a diversas circunstancias, que van desde lo académico hasta lo social, es necesario postular una orientación marcada: la enseñanza de la escritura no se debe solo al aula de clases. Esta pasa por diversas formas de agenciar el conocimiento, involucra actores más allá de profesores y tutores, se proyecta hacia fines que no se agotan en las necesidades y en las demandas académicas. North (1984) los describe como espacios que los escritores utilizan para hablar sobre la escritura. Esta definición, plantean Gavari y Tenca (2017), supone situar en primer lugar a las personas, los estudiantes escritores, otorgando ya una idea sutil de dónde se ubica el foco de atención y el proceso de transformación. Asimismo, continúan, se intuye un aspecto dialógico y de interacción entre los actores que pasan por el proceso de escritura.

El Cleo de la UdeA mira más allá de las necesidades del estudiante in situ en su relación con las prácticas letradas en el seno de la universidad. Centra su mirada en el proceso de leer y escribir, ya que parte del principio de que este, más que un afianzamiento de habilidades y competencias, da cuenta de unas prácticas que responden a contextos, realidades e historias de los sujetos que convergen en la universidad.
Sin embargo, compartimos con los centros de escritura la promoción del aprendizaje colaborativo y la construcción social del conocimiento, a través de una relación dialógica con el otro. Esto quiere decir que, en el acompañamiento que hacemos, reconocemos una disposición de saberes por parte nuestra, y permitimos, al tiempo, que el tutorado nos enseñe la forma en que lee, escribe y comprende.

De igual manera, Zanotto González y sus colegas (2016) mencionan las que pueden considerarse acciones alternas realizadas en los centros de escritura (CE). Esta lista comprende:

- Centros de entrenamiento o capacitación para los tutores. En muchos de los centros, los tutores son estudiantes de pregrado o de posgrado, situación que reclama un espacio de capacitación, no necesariamente de formación, que implica un proceso distinto.

- Investigación. Los CE ofrecen una amplia base investigativa en prácticas empíricas o basadas en evidencia (Babcok y Thonus, como se cita en Zanotto y González, 2016), que han contribuido a la consolidación de una comunidad académica cada vez más desarrollada.

- Acompañamiento y formación de docentes. A partir de la escritura a través del currículo, la relación entre el centro y los cursos se estrecha para pensar de manera conjunta las lógicas de producción académica de los estudiantes.

- Producción de materiales y recursos educativos para el desarrollo de las competencias de lectura y escritura. Aprovechando el potencial de los recursos informáticos en asocio con las prácticas de lectura y de escritura es posible descentrar los encuentros sincrónicos hacia los asincrónicos.

Como se verá en el siguiente apartado, algunas de las acciones que se describen como propias de los centros de escritura están presentes en el CLEO, pues somos conscientes de que el trabajo con los estudiantes puede derivar en una problematización 
de las prácticas (uno de los propósitos de la literacidad académica), pero un trabajo que involucre a los maestros y a otros actores de las dinámicas universitarias puede tener un mayor alcance, cuando afecta a los programas mismos.

\section{LEO en la práctica: las estrategias de formación del CLEO, UdeA}

Tras el recorrido que presentamos en las páginas anteriores, podemos afirmar que contamos con el marco conceptual propicio para pensar las LEO como prácticas dialógicas en el contexto de la educación superior y desde la configuración del espacio institucional del CLEO; por tanto, en el presente apartado, mostramos el funcionamiento de tres estrategias formativo-pedagógicas en las cuales se sustenta el trabajo del CLEO desde el año 2018, una vez pasada la fase de diseño e iniciada la implementación, en la cual se pone en marcha el dispositivo desde la definición de sus enfoques y el trabajo con los diferentes estamentos de la comunidad universitaria (docentes, estudiantes y administrativos). Son, entonces, estas líneas las que permiten establecer una conversación entre los enfoques didáctico-educativos de la alfabetización académica, y los enfoques discursivo-etnográficos del campo de la literacidad académica.
En síntesis, nos parece importante reflexionar sobre este ejercicio formativo que pretende generar una práctica coherente con su filosofía de enseñanza y lenguaje. La Figura 3 nos ofrece información acerca de la estructuración interna del CLEO, resaltando las líneas que constituyen el CLEO - gestión, acompañamiento y formación-, y las acciones derivadas de cada una de estas, desde el ejercicio horizontal de coordinación colegiada que hemos propuesto e implementado para su funcionamiento.

A partir de la ruta esbozada aquí, a continuación, delineamos a grandes rasgos una muestra de las estrategias desarrolladas en cada una de líneas de acción configuradas en el CLEO, para mostrar, desde allí, la manera como venimos articulando las visiones de las prácticas LEO, la formación institucional y la permanencia.

Para este ejercicio, en la línea de gestión, destacamos el espacio del Café CLEO, como una propuesta de difusión del centro, dirigida a los diferentes estamentos de la comunidad universitaria. En la línea de acompañamiento, mostramos la estrategia Tallereando con CLEO, mediante la que se ofrecen talleres para estudiantes pertenecientes a las diferentes unidades académicas de la sede

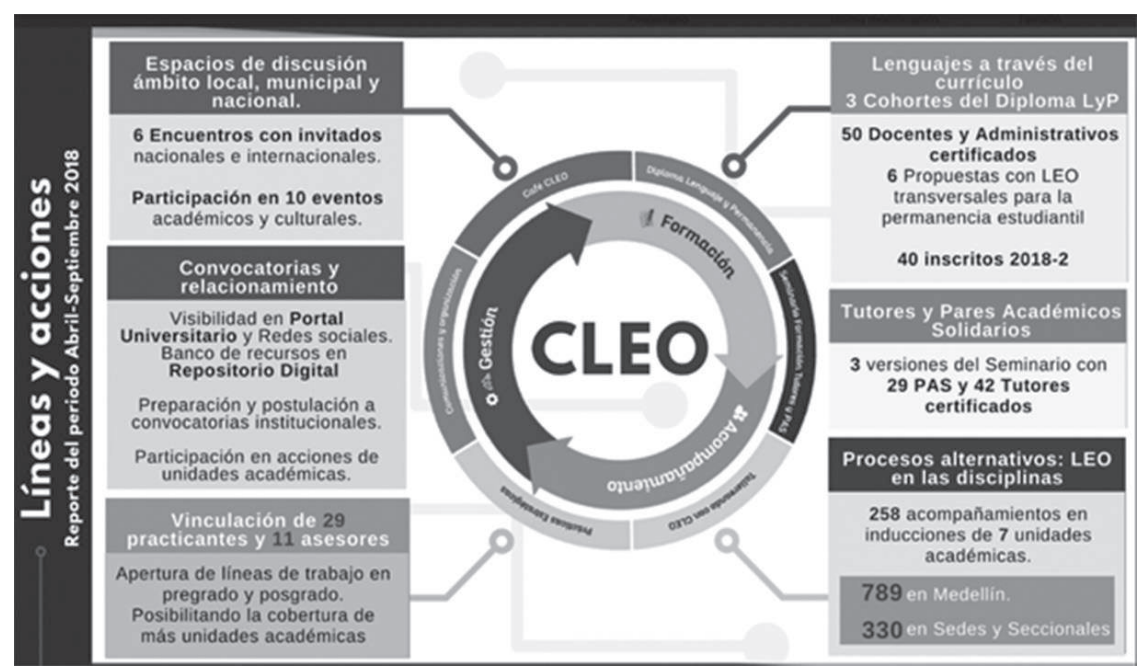

Figura 3 Estructura, líneas y acciones del CLEO. 
central y de regiones, algunos de quienes son pares tutores solidarios. Por último, en la línea de formación, abordamos el espacio del Diploma Lenguaje y Permanencia, atendido por medio del programa de formación docente de la universidad, a todos los maestros (vinculados, ocasionales y cátedra) y empleados administrativos.

\section{Estrategia de formación de la línea de gestión: Café CLEO}

El Café Cleo es una estrategia de difusión de las discusiones sobre las prácticas LEO; se constituye en un espacio para consolidar la comunidad académica en torno al tema. Este espacio es asumido por el colectivo como un espacio mensual de conversación abierta con diferentes estamentos universitarios, pues son convocados estudiantes, docentes e invitados de la comunidad, para conversar en un ambiente distendido sobre temas de actualidad para las prácticas LEO en educación superior. Estas conversaciones tienen una duración de 2 horas, durante las cuales se plantean reflexiones y líneas de fuga para seguir pensando estrategias de formación y acompañamiento acorde a las necesidades e intereses identificados en la comunidad.

Así las cosas, durante 2018 se han llevado a cabo nueve espacios de Café CLEO, liderados por invitados locales, nacionales e internacionales, con amplia trayectoria y experiencia en aspectos clave para el desarrollo del centro en el marco de eventos institucionales. Las temáticas abordadas por los invitados, con la moderación de la línea de gestión del CLEO, fueron: 1) apuestas políticas y justicia social en Brasil y Colombia, 2) economía del bien común, 3) currículo y lenguaje, 4) memoria, lenguaje y biblioteca, 5) lenguaje y formación de maestros, 6) acompañamiento en las prácticas LEO, 7) literatura para bebés, 8) literacidades e interculturalidad y 9) proyectos interculturales en salud y epidemiología con enfoque social.

El Café CLEO es una apuesta por configurar diálogos y alianzas intra- e inter- institucionales que instalen las preguntas por el lenguaje, las disciplinas y la permanencia en la agenda de los diferentes estamentos, para, de esta forma, posibilitar reconocernos, gestionar nuevos marcos conceptuales en torno a las prácticas LEO y vincularlas con las maneras como los sujetos habitan cotidianamente las aulas universitarias, los contextos profesionales, la ciudad y la vida misma.

Tal y como lo hemos mencionado en algunas partes del texto, la postura teórica del CLEO permite repensar de manera constante las relaciones entre los sujetos y las prácticas discursivas. Visto así, es una pretensión que abre todo un panorama muy variado de posibilidades en las cuales entran manifestaciones de producción, y de comprensión. De allí que desde la gestión se busque dar cabida a horizontes de comprensión, ora cercanos, ora alejados de nuestras concepciones. Esto guarda relación con la idea que esbozan Zanotto González et al. (2016), cuando dicen

Uno de los principales errores al implementar este tipo de programas (centros de escritura) es asumir que después del montaje inicial ya se resolvió la necesidad que motivó su apertura y solo hay que mantener la propuesta inicial. Por el contrario, la socialización constante con los usuarios, a través de campañas publicitarias de diversa índole, y con los administrativos, a través de informes bien elaborados, renueva la vinculación. (p. 353)

Una comprensión amplia de las formas como se produce lenguaje en la universidad requiere a su vez contar con concepciones variadas sobre el tema. Lo anterior nos permite, a un tiempo, contrastar posturas, prácticas y creencias acerca de la escritura, al tiempo que configuramos un centro de nueva generación, que pueda pluralizar su visión de lenguaje y orientarse a perspectivas interculturales, al tiempo que considere la oralidad en sus múltiples y diversas ocurrencias.

\section{Estrategia de la línea de acompañamiento: Tallereando con CLEO}

La estrategia de Tallereando con CLEO busca dinamizar recursos didácticos que aportan a la 
apropiación de las prácticas LEO, con base en las necesidades e intereses formativos para el aprendizaje, en la cual se involucra a estudiantes y tutores. Esta estrategia es un aporte al mejoramiento de las prácticas académicas, y en general universitarias, y que, por tanto, ayuda a la permanencia y graduación de los estudiantes. Así las cosas, el CLEO destaca la importancia de llevar a cabo, con las diferentes áreas de conocimiento, un acompañamiento formativo (tutorías) que favorezca la apropiación de las prácticas de lectura, escritura y oralidad, en relación con las comprensiones que se entretejen y configuran desde la universidad y que atraviesan las prácticas no solo académicas, sino también sociales e individuales de aquellos que la conforman y le dan vida.

Esta estrategia tiene como base un repositorio virtual de configuraciones didácticas ${ }^{8}$ basadas en talleres por trayectos de formación; de este modo se plantean tres tipos de talleres estructurados, desde los planteamientos de Litwin (2012), para darle un sentido a las prácticas, ya que se direccionan por unos objetivos particulares vinculados a la enseñanza-aprendizaje en relación con unas necesidades que "vinculan unos saberes y saberhacer, en el marco de una situación discursiva que le otorga sentido" (Pérez y Rincón, 2009, p. 19). Estos talleres fueron inspirados en propuestas que se desarrollaron en experiencias de las prácticas pedagógicas que dieron base para el centro. Para este propósito, los tres tipos de talleres implementados en el CLEO se clasifican de la siguiente manera:

- Talleres de Recibo y acogida a la vida universitaria, que en algunos casos se ofertan en alianza con la Dirección de Bienestar Universitario durante las jornadas de inducción a los estudiantes de nuevo ingreso. Estos talleres tienen una duración de dos horas y van desde los talleres que indagan por la biografía lectora de los

8 Página del repositorio virtual: http://www.udea. edu.co/wps/portal/udea/web/inicio/institucional/ permanencia-estudiantil/iniciativas/cleo participantes, pasando por el taller titulado ¿Para qué citar? y el taller de Escritura creativa. En este punto destacamos el trasfondo de alfabetización académica de esta iniciativa, pues, como lo considera Carlino (2005), el desempeño en lectura y escritura de los estudiantes es una responsabilidad compartida entre ellos y los maestros.

- Talleres de vinculación y saberes disciplinares, que se ofrecen en las unidades académicas con énfasis en los diferentes lenguajes asociados con las disciplinas y saberes propios de los currículos específicos de los programas de formación. Algunos de estos tienen duración de dos horas y otros se componen de diversas sesiones de trabajo, que van de dos a ocho encuentros. A este tipo de talleres corresponden: Pasaporte a la cultura académica, Leo en inglés, Literatura y escritura en la U: preparación de presentaciones orales, Literatura científica: tipos de razonamiento y FLISCLEO: Lenguajes para la permanencia (inglés y español con estudiantes de comunidades étnicas); con énfasis en lengua castellana, lengua inglesa, lenguajes matemáticos y lenguas ancestrales.

- Y, por último, tenemos los talleres de trabajo autónomo, graduación y proyección, que tienen un tiempo de duración variable, de acuerdo con el proceso que se acompaña; estos cuentan con un espacio de acompañamiento tutorial para la escritura de trabajos de grado, y para la elaboración y presentación de proyectos desde las estrategias de argumentación, cuya duración está entre dos y cuatro sesiones.

La participación en los talleres es gestionada directamente por los estudiantes interesados o por maestros que quieren acompañar y potenciar las prácticas LEO de los participantes en sus cursos y asumen el proceso en calidad de tutores, lo cual da cuenta de un proceso de reconocimiento de las acciones CLEO en diferentes ámbitos y 


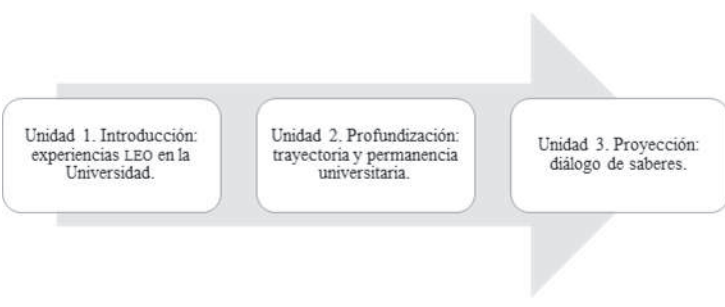

Figura 4 Unidades del diploma en Lenguaje y Permanencia.

estamentos de la vida universitaria. Esta iniciativa se orienta a través de las disposiciones de la alfabetización académica, por cuanto busca un enfoque más didáctico-educativo y aborda más las acciones institucionales y didácticas de la enseñanza-aprendizaje en las disciplinas o campos de saber; así como de la literacidad académica, por cuanto busca por medio del diseño de sus talleres reconocer la trayectoria universitaria y la biografía de los estudiantes que llegan a la institución con el fin de poner en negociación las diferentes prácticas letradas.

\section{Estrategia de la línea de formación: diploma Lenguaje y Permanencia}

En los aspectos metodológicos, se destaca su modalidad semipresencial, con dos espacios: virtual y presencial. El espacio virtual está constituido por 70 horas y el presencial por 20, y es organizado en tres unidades. Cada una de las unidades de este Seminario-taller se orienta a partir de las siguientes preguntas: ¿cuál es la relación entre lectura, escritura y oralidad académicas con la permanencia estudiantil?, ¿cómo potenciar las prácticas LEO para evitar la deserción estudiantil por motivos académicos? Teniendo presentes dichas preguntas, las unidades están diseñadas de modo que cada subtema profundice en aspectos implicados en el lenguaje académico y permanencia estudiantil. La Figura 4 expone las unidades en las que se ha dividido el diploma.

Dentro del espacio virtual se buscan generar reflexiones a partir de documentos e investigaciones sobre lectura y escritura en la universidad, así como foros de discusión donde se pondrán en común ejercicios y aprendizajes en torno a los temas de cada unidad. En el espacio presencial, se busca propiciar un reconocimiento de los participantes, de modo que el diálogo de persona a persona propicie la comprensión de la práctica docente en la universidad. La producción final de este diploma tiene como meta provocar incidencia en la transformación curricular en los programas de la Universidad.

Acorde a este propósito, acompañamos procesos de rediseño de programas de curso o elaboración de nuevos espacios de formación y proyectos, todos con el reto de explicitar su vínculo con las prácticas de lectura, escritura y oralidad en las disciplinas. Esta producción debe proponerse a incorporar una visión de las prácticas LEO que intente descolonizar las concepciones hegemónicas de lenguaje, propósito que se alinea con un enfoque discursivo-etnográfico (Zavala, 2010) con el cual reconocer los contextos diversos de producción de saber de los estudiantes.

El diploma también se orienta por principios de la alfabetización académica, cuando se toma el tema de las prácticas LEO como algo institucional, y diseña una estrategia transversal de formación de docentes y administrativos. Dentro de dicha reflexión, reconocemos que el papel del maestro será fundamental para transformar las prácticas de lectura, escritura y oralidad en los estudiantes de pregrado. Ello quiere decir que es necesario dilucidar cómo se lee y escribe en cada campo disciplinar, para crear conciencia en los estudiantes de las particularidades de su área de estudio y explicitar la doble tarea que le implica la formación profesional, para así evitar la deserción estudiantil por motivos académicos.

\section{Enlace entre las estrategias CLEO}

$\mathrm{Al}$ presentar esta experiencia pedagógica, a partir del funcionamiento de tres estrategias formativopedagógicas en las cuales se sustenta el trabajo del CLEO, nos propusimos a explicitar sus enfoques y 
diseño, para poner en discusión los alcances que han tenido en diferentes actores de la comunidad universitaria (docentes, estudiantes y administrativos) y así posicionar como discusión y como reflexión el papel del lenguaje en la vida académica. Además, mostramos cómo estas estrategias contribuyen de diferentes formas al proceso formativo de los estudiantes universitarios, a su permanencia y a su graduación exitosa.

En síntesis, desde el enfoque de alfabetización académica, las estrategias formativo-didácticas presentadas retoman la idea de que para la comprensión de cada uno de los campos de saber es necesario el reconocimiento de su discurso, así como las formas de interpretarlo, tarea en la que es menester la participación de los maestros, de los educandos y del personal administrativo de la universidad, en quienes recae el compromiso de la institucionalización de las prácticas y de los enfoques; todos ellos terminan adelantando procesos de apropiación de las convenciones del lenguaje académico.

Desde el enfoque de literacidad académica, las estrategias formativo-didácticas presentadas retoman una mirada problemática de las prácticas discursivas, en las cuales importa no solo el fin, el para qué, sino también los medios de producción del discurso. En otras palabras, desde posturas críticas (enfoques de pensamiento decoloniales, de plurilingüismo, diversidad cultural y lingüística aplicada, reconocimiento del otro), se intenta poner en tensión las formas de usar el lenguaje en la Universidad, así como la visibilización y el reconocimiento de las prácticas vernáculas para ponerlas en diálogo con las convenciones institucionales (del poder, el local-global).

Luego de esta discusión y reflexión sobre las estrategias, nos parece importante señalar que quizá este centro haga parte de una segunda generación de centros de lectura y escritura, al pluralizar su visión de lenguaje y orientarse a perspectivas interculturales, al tiempo que considera la oralidad en sus múltiples y diversas ocurrencias.

\section{Consideraciones finales}

La revisión de los componentes en los que está fundamentado el CLEO de la Universidad de Antioquia testimonia el lugar preponderante que este tipo de centros tiene en la educación universitaria para las diversas comunidades que participan en ella. En un lugar muy central se ubican los enfoques de la literacidad académica y la alfabetización académica, y se trabaja, desde sus iniciativas, para ir reconfigurando la pregunta por la pluralidad de lenguajes que habitan la universidad, representados por poblaciones cuyas trayectorias de vida terminan por hacerse visibles en las formas de comunicar.

A partir de esta lógica, el CLEO se propone el acompañamiento tutorial, pero incursiona también en la formación de un público (estudiantes, profesores, administrativos), que pueda garantizar movilidad académica que incida en la permanencia y la graduación exitosa de los estudiantes. No nos detenemos en acciones remediales (aunque no las subvaloramos), toda vez que asumimos la lectura, la escritura y la oralidad como prácticas que se entienden a partir de un contexto de producción (literacidad académica).

Lo anterior nos presenta una cuestión que intentamos problematizar: el desempeño académico de los estudiantes a menudo guarda estrecha relación con su condición social ${ }^{9}$. Esta es una de las razones por las cuales nos configuramos como un centro de nueva generación, en el que la interculturalidad nos dé pistas para comprender la diversidad de sujetos que viven la universidad. Para ello buscamos:

- La formación, con la que se busca resonar, desde el enfoque de la literacidad académica y la alfabetización académica, maneras alternas de relacionarse con el lenguaje, bien sea

9 Vale la pena recordar que el $85 \%$ de la población de los estudiantes de la UdeA pertenece a los estratos 1, 2 y 3 (UdeA, 2017). 
desde el ámbito docente, bien sea desde las interacciones que ocurren en otros espacios de relación distintos del salón de clases.

- El acompañamiento, que establece una relación dialógica con los estudiantes tanto de la ciudad de Medellín como de las subregiones del departamento de Antioquia. Esta línea es, de manera práctica, la que desarrolla de manera más justa el principio vital del CLEO, pues es en el encuentro con el otro que nuestro trabajo encuentra el sentido de ser, de saber y de hacer.

- La gestión, en la cual se establecen los contactos con los demás actores estratégicos que a su manera vienen pensando sobre lo pensado acerca del lenguaje en la universidad. Estos diálogos son los que nos han permitido hacer a un lado las certezas en las que tan fácilmente se cae cuando se piensa en la comunicación.

- El diálogo intersectorial e interdisciplinar promovido en los diálogos con unidades académicas y administrativas.

Las estrategias vistas en el tercer apartado, aunque plausibles y bien intencionadas, no están exentas de inquietudes. Ocurre muchas veces con la alfabetización, por poner un ejemplo. Se piensa que cuando el estudiante aprende a hablar la lengua especializada que propone la universidad ha superado entonces la demanda que hace la comunidad académica. Estas estrategias reflejan un quehacer instrumental más que funcional; mecanizante más que reflexivo. Este ejemplo no hace más que seguir presentando inquietudes que nos obligan a no olvidar todos los elementos a considerar en el trabajo que día a día hacemos.

Es un interés del CLEO la generación de un diálogo entre los enfoques y los modelos de manera que sitúan a los estudiantes en una posición desde la que se reconocen productores de prácticas LEO en la universidad y que puedan negociar sus prácticas letradas, como nos proponen Lea y Street
(1998) con el modelo de literacidades, lo cual destaca la necesidad de continuar poniéndolos en tensión desde espacios académicos abiertos que habiliten la conversación y el reconocimiento de la pluralidad.

El diploma Lenguaje y Permanencia fue diseñado para generar un espacio de formación que propicie la reflexión sobre la importancia de la relación entre el lenguaje académico y la permanencia estudiantil con docentes de diferentes áreas de conocimiento de la Universidad. Su principal objetivo es promover la visibilización sobre el proceso de apropiación de las prácticas discursivas de lecturas, escrituras y oralidades de las disciplinas específicas que sustentan los programas de formación de la Universidad, con el fin de fortalecer el ingreso, permanencia y graduación de los estudiantes.

A pesar de lo logrado, es necesario seguir apostándole en el CLEO al reconocimiento de los avances que, en materia de educación para el desarrollo, la interculturalidad y las estrategias de inclusión académica, viene haciendo la universidad en Colombia, y particularmente la Universidad de Antioquia. Este es un rasgo distintivo del CLEO respecto de otros centros regionales y nacionales, así como su estructura organizativa horizontal, vinculada con una coordinación colegiada que se constituye en una apuesta epistemológica desde donde se ponen en tensión las configuraciones de saber-poder que le dan fuerza a su consolidación como comunidad de aprendizaje que convoca la mayor cantidad y diversidad de unidades académicas y no solo a aquellas a las que tradicionalmente se les ha endilgado y encargado la "tarea" de la lectura, la escritura y la oralidad.

Este escenario da cuenta de un pensar lo pensado, en el sentido de crear las condiciones para un verdadero diálogo cultural que se materialice en la práctica. Una integración que se refleje en la vida de la cotidianidad, en las acciones mínimas que cualquier ser humano emprende día a día. Lo 
que se ha hecho nos hacen pensar que las apuestas que se hacen están orientadas en la dirección adecuada.

Por último, queremos enunciar los retos que se nos presentan en el mediano y en el largo plazo:

- Para el crecimiento del centro en términos de articulaciones y diálogos, es imperativo seguir pensando en nuevas formas de manifestaciones del lenguaje que se puedan integrar a la reflexión de aquello que este hace posible, en términos de la vida cotidiana y la pertenencia a una comunidad académica.

- Hay una necesidad latente frente a la generación de espacios alternos o no convencionales por donde pueda tener ocurrencia el CLEO, en tal sentido habrá que preguntarse por ¿Cómo avanzar en pensar algo más que un aula para el centro?

- El CLEO debe seguir construyendo la idea de situarse como un centro de segunda generación. Para ello, se hace interesante mapear las relaciones inter y transdisciplinares, los lenguajes multimodales, las nuevas formas de subjetivar saberes y disciplinas en el contexto académico universitario, en la ciudad y en las regiones (en donde venimos adelantando acciones que cada vez son más demandadas por las comunidades), de modo que se autoricen nuevas preguntas para las prácticas LEO en educación superior.

- Es pertinente que el CLEO comience a pensar otras formas, adicionales a la literacidad académica, para abordar el asunto de las prácticas LEO en la Universidad. Así las cosas, este se configura como un campo teórico en tensión desde el cual se puede aportar sustancialmente, si consigue equilibrar las fuerzas presentes en sus tres líneas: gestión, acompañamiento y formación Las preguntas que quedan son ¿Será posible hacer un quiebre teórico al enfoque sociocultural cuando algunos centros aún transitan por una mirada asistencial y enfocada en los aspectos lingüísticos para el abordaje de las LEO?, ¿De qué manera la Universidad de Antioquia puede aportar para la discusión en torno a las prácticas LEO, desde un trabajo de articulación entre docencia, investigación y extensión?

- Finalmente, se hace necesario explicitar las visiones de tutoría que circulan en la universidad, en diálogo con la apuesta que viene configurando el CLEO, desde estrategias como el Seminario de formación de tutores y pares académicos solidarios e incluso desde el mismo Diploma Lenguaje y Permanencia, en paralelo con las nuevas concepciones del lenguaje y las literacidades.

\section{Referencias}

Bazerman, C., Little, J., Bethel, L., Chavkin, T., Fouquette, D. y Garufis, J. (2016). Escribir a través del curriculum. Una guia de referencia. (Federico Navarro, editor). Córdoba: Universidad Nacional de Córdoba.

Carlino, P. (2002). ¿Quién debe ocuparse de enseñar a leer y a escribir en la universidad? Lectura y vida, 23(1), 6-14.

Carlino, P. (2003). Leer textos científicos y académicos en la educación superior: obstáculos y bienvenidas a una cultura nueva. Uni-pluri/versidad, 3(2), 17-23.

Carlino, P. (2005). Escribir, leer y aprender en la universidad. Una introducción a la alfabetización académica. Buenos Aires: Fondo de Cultura Económica de Argentina.

Carlino, P. (2013). Alfabetización académica diez años después. Revista Mexicana de Investigación Educativa, 18(57), 355-381.

Chalmers, D. y Fuller, R. (1996). Teaching for Learning at University. Londres: Kogan Page.

Freire, A. M. A. (1989). Analfabetismo no Brasil: da ideologia da interdição do corpo à ideologia nacionalista, ou de como deixar sem ler e escrever desde Catarinas (Paraguaçu), Filipas, Madalenas, Anãs, Genebras, Apolônias e Gracias até os Severinos. São Paulo: Cortez, Brasília, DF: INEP.

Freire, P. (1996). Pedagogia da autonomia: saberes necessários à prática educativa. São Paulo: Paz e Terra.

Freire, P. (2012[1970]). Pedagogía del oprimido. Madrid: Biblioteca Nueva. 
Gavari, E. y Tenca, P. (2017). La evolución histórica de los centros de escritura académica. Revista de Educación, (378), 9-29.

Heath, S. B. (1982). What no bedtime story means: narrative skills at home and school. Language and Society, (11), 49-76.

Hornberger, N. (2001). Criando contextos eficazes de aprendizagem para o letramento bilíngue. En M. I. Cox y Z. Z. Assis-Peterson (Orgs.), Cenas de sala de aula. Campinas, SP: Mercado de Letras.

Kalman, J. y Street, B. (org.). (2009). Lectura, escritura y matemáticas como prácticas sociales. Diálogos con América Latina. México: Siglo XXI.

Kleiman, A. B. (org.) (1995). Os significados do letramento: uma nova perspectiva sobre a prática social da escrita. Campinas, SP: Mercado Aberto.

Kleiman, A. B. y Sito, L. (2016). Multiletramentos, interdições e marginalidades. En A. B. Kleiman y J. Assis, (Orgs.), Significados e ressignificaçôes do letramento. Desdobramentos de uma perspectiva sociocultural sobre a escrita (pp.169-198). Campinas, sp: Mercado de Letras.

Kleiman, A. B.; Vianna, C. y De grande, P. (en preparación). A iniciação científica como prática social: desvendando os "mistérios" do letramento acadêmico na licenciatura. En J. G. Moura y M. A L. Rossi (Orgs.), A iniciação cientifica nas licenciaturas: a pesquisa como prática de formação de professores. Goiânia: UFG.

Ladson-Billings, G. (2008). Os guardióes de sonhos. O ensino bem-sucedido de crianças afro-americanas. Belo $\mathrm{Ho}-$ rizonte: Autêntica.

Lea, M. R. y Street, B. (1998). Student writing in higher education: an academic literacies approach. Studies in Higher Education, 23(2), 157-173.

Lillis, T. (1999). Whose "common sense"? Essayist literacy and the institutional practice of mystery. En C. Jones, J. Turner, y B. Street, (orgs.), Students writing in the university: cultural and epistemological issues. Amsterdam: John Benjamins Publishing.

Litwin, E. (2012). El oficio de enseñar, condiciones y contextos. Barcelona: Paidós

Moreno Mosquera, E. (2019). Lectura académica en la formación universitaria: tendencias en investigación. Lenguaje, 47(1), 91-119. doi: 10.25100/ lenguaje.v47i1.7180

Moje, E. (2008). Foregrounding the disciplines in secondary literacy teaching and learning: A call for change. Journal of Adolescent \& Adult Literacy, 52(2), 96-107.
Narváez, E. y Cadena, S. (orgs.). (2008). Los desafíos de la lectura y la escritura en la educación superior: caminos posibles. Cali: Universidad Autónoma de Occidente.

Navarro, F., Ávila Reyes, N., Tapia-Ladino, M., Cristovão, Vera L. L, Moritz, M. W, Narváez Cardona, E, y Bazerman, C. (2016). A historical and contrastive account of higher education reading and writing studies published in Latin America. Revista Signos, 49(Supl. 1), 78-99. https://dx.doi.org/10.4067/ S0718-09342016000400006

North, S. M. (1984). The idea of a writing center. College English, 46(5), 433-446.

Pérez, M. y Rincón, G. (2009). Actividad, secuencia didáctica y pedagogía por proyectos: tres alternativas para la organización del trabajo didáctico en el campo del lenguaje (pp. 1-43). CERLALC. Disponible en https:// scf563291e8cb4ead.jimcontent.com/download/ version/1434142807/module/8404496383/name/_ Alternativas\%20RED\%20DE\%20LENGUAJE.pdf

Pérez, M. y Rincón, G. (2013). ¿Para qué se lee y se escribe en la universidad colombiana? Un aporte a la consolidación de la cultura académica del pais. Bogotá: Editorial Pontifícia Universidad Javeriana.

Sito, L. (2016). Escritas afirmativas: estratégias criativas para subverter a colonialidade em trajetórias de letramento acadêmico. Tesis de doctorado en Lingüística Aplicada, Instituto de Estudos da Linguagem, Universidade Estadual de Campinas, Campinas, SP.

Sito, L. y Kleiman, A. B. (2017). "Eso no es lo mío”: un análisis de conflictos en la apropiación de prácticas de literacidad académica. Universitas Humanistica, 83, 153-179. http://dx.doi.org/10.11144/Javeriana.uh83.acap

Soler, S. (2013). Usted ya en la universidad y no saber escribir. Escritura y poder en la universidad. Bogotá: Universidad Distrital Francisco José de Caldas.

Soler, S. y Pardo, N. (2007). Discurso y racismo en Colombia. Cinco siglos de invisibilidad y exclusión (pp.181-227). Racismo y discurso en América Latina. Barcelona: Gedisa.

Street, B. (1993). The New Literacy Studies. En B. Street (org.), Cross-cultural approaches to literacy. Cambridge: Cambridge University Press.

Street, B. (2010). Dimensões "escondidas" na escrita de artigos acadêmicos. Perspectiva, 28(2), 541-567. Florianópolis. Disponível em: http://www.periodicos.ufsc.br/index.php/perspectiva/article/ view/2175-795X.2010v28n2p541/pdf.

Universidad de Antioquia -UdeA-. (2015). Misión. Direccionamiento estratégico. Medellín. Disponible en 
http://www.udea.edu.co/wps/portal/udea/web/ inicio/institucional/direccionamiento-estrategico

Universidad de Antioquia -UdeA- (2017, 4 diciembre). UdeA recibe a 5.467 estudiantes. Periódico Alma Mater. Recuperado de https://goo.gl/oMM9pj

Vargas Franco, A. (2015a). Escribir en la universidad. Reflexiones y estrategias sobre el proceso de composición escrita de textos académicos. Cali: Editorial Universidad del Valle.

Vargas Franco, A. (2015b). Literacidad crítica y literacidades digitales: ¿una relación necesaria? Una aproximación a un marco teórico para la lectura crítica. Revista Folios, (42), 139-160, julio-diciembre.

Zanotto González, M. y Perales, M. D. (2016). Enseñar a leer y escribir en la educación superior. Propuestas educativas basadas en la investigación. Tamaulipas: 2think Design Studio. Disponible en http://web. metro.inter.edu/facultad/esthumanisticos/crem docs/Ensenar\%20a\%20leer\%20y\%20escribir.pdf
Zavala, V. (2010). Quem está dizendo isso?: letramento acadêmico, identidade e poder no ensino superior. En: C. L. Vóvio, L. S. Sito e P. B. De Grande, Letramentos: rupturas, deslocamentos e repercussóes de pesquisas em linguistica aplicada (pp. 71-95). Campinas, SP: Mercado de Letras.

Zavala, V. (2011). La escritura académica y la agencia de los sujetos. Cuaderno comillas, (1), 52-66.

Zavala, V. y Córdova, G. (org.). (2010). Decir y callar. Lenguaje, equidad y poder en la universidad peruana. Lima: Editorial de la Pontificia Universidad Católica del Perú.

Zavala, V.; Niño-Murcia, M.; Ames, P. (eds.). (2004). Escritura y sociedad. Nuevas perspectivas teóricas y etnográficas. Lima: Red para el Desarrollo de las Ciencias Sociales en el Perú.

How to reference this article: Sito, L.; Méndez-Rendón, J. C. y Vásquez-Ramírez, L. Y. (2019). LEO en la práctica: la experiencia formativa en un centro de lecturas, escrituras y oralidades. Íkala, Revista de Lenguaje y Cultura, 24(2), 419-438. doi: 10.17533/udea.ikala.v24n02a12 